\title{
Calibration and Monte Carlo Pricing of the SABR-Hull-White Model for Long-Maturity Equity Derivatives
}

\author{
Bin Chen, Lech A. Grzelak ${ }^{\dagger}$ and Cornelis W. Oosterlee ${ }^{\ddagger}$
}

this version: December 7, 2011

\begin{abstract}
We model the joint dynamics of stock and interest rate by a hybrid SABR-HullWhite model, in which the asset price dynamics are modeled by the SABR model [18] and the interest rate dynamics by the Hull-White short-rate model [19]. We propose a projection formula, mapping the SABR-HW model parameters onto the parameters of the nearest SABR model. Further a time-dependent parameter extension of this SABR-HW model is introduced to make the calibration of the model consistent across maturities. The inverse of the projection formula enables a rapid calibration of the model. As the calibration quality subjects to the approximation errors of the projection formula, we subsequently apply a non-parametric numerical calibration technique based on the non-uniformly weighted Monte Carlo technique [5] to improve the calibration. In this step, the Monte Carlo weights are not uniform and chosen in such a way that the calibration market instruments are perfectly replicated.
\end{abstract}

Key words: Stochastic volatility; hybrid SABR-HW model; calibration; model approximation; timedependent parameters; weighted Monte Carlo method; low-bias Monte Carlo simulation.

\section{Introduction}

Equity derivative models and yield curve models have been developed independent of each other for a long time. Whereas the equity derivative models have been focused on the implied volatility skew/smile by local or stochastic volatility features [13], short-rate models improved the accuracy of the yield curve dynamics. With an increasing interest in long-maturity equity derivatives, as well as in equity-interest rate hybrid products from retail and long term institutional investors (see [20]), the industrial practice also demands models that are capable of describing joint dynamics of interest rates and equity. Indeed, one would intuitively assert that the interest rate is stochastic and that there is non-zero correlation between the interest rate and the equity.

In the present paper, we propose a hybrid extension of the Stochastic Alpha Beta Rho (SABR) model [18] to model the joint equity-interest rate dynamics. We construct a hybrid model, called the SABR-Hull-White (SABR-HW) model, in which the equity process is driven by the SABR model and the interest rate by the short-rate model of Hull-White [19]. In this framework the equity process is assumed to be correlated with the interest rate process.

\footnotetext{
${ }^{*}$ Corresponding author. CWI - National Research Institute for Mathematics and Computer Science, P.O. Box 94079, 1090 GB, Amsterdam and Rabobank International, Utrecht, the Netherlands. Email: B. Chen@cwi.nl

${ }^{+} \mathrm{CWI}$ - National Research Institute for Mathematics and Computer Science, Amsterdam and Rabobank International, Utrecht. E-mail address: L. A.Grzelak@cwi.nl.

${ }_{\text {} C W I}$ - National Research Institute for Mathematics and Computer Science, Amsterdam and Delft University of Technology, Delft Institute of Applied Mathematics, The Netherlands. E-mail address: C.W.Oosterlee@cwi.nl.
} 
The SABR model, which is not often used in the equity derivative literature, has several attractive features for the modeling of long term equity-linked products. It generalizes stochastic volatility models such as Heston's model by introducing an explicit stock price dependence in a power law local volatility term, $f(x)=x^{\beta}$ [23]. Further, the SABR model admits a closed-form approximation formula ("Hagan's formula") for the Black implied volatilities which greatly simplifies calibration. Thirdly, the SABR process is, for certain parameter values, an absorbing process at the zero asset price boundary [11], which models the fact that companies may default in time ([23]). Last but not least, the parameters in the SABR model have a direct connection to market instruments or market price features, in contrast to, for example, the speed-of-meanreversion parameter in the Heston model.

One contribution in this paper is an invertible projection formula of the constant parameter SABR-HW model onto the plain SABR model. This formula enables a highly efficient calibration of the constant parameter SABR-HW model based on the established calibration procedure for the SABR model. The resulting calibration parameters remain however only valid for a single maturity time and cannot provide a consistent dynamic description of the underlying asset prices across multiple maturity times. We deal with this issue by adopting a dynamical SABR(-HW) extension in the spirit of Rebonato [26].

Moreover, we will use the well-known weighted Monte Carlo (WMC) technique, proposed by Avellaneda et al. [5], as another stage of calibration of the SABR-HW model. By this we can deal with the inconsistency between the true model dynamics and those implied by Hagan's asymptotic approximation formula (by which the calibration instruments are quoted).

For the Monte Carlo simulation, we adopt a low-bias discretization for the SABRHW dynamics, which has some advantages over a basic Euler scheme as it gives a low bias (i.e. stable and accurate) when large time steps are used (say 4 time steps per year), see [11].

This paper is organized as follows. In Section 2, we define the dynamical SABRHW model and discuss the building blocks. We show how to project the constant parameter SABR-HW dynamics onto a plain SABR model in Section 3. In Section 4, we utilize this projection formula to calibrate the constant parameter version of the model. We also show how to determine the time-dependent functions in the dynamical SABRHW model and use the WMC technique [5]. In Section 5, the low-bias Monte Carlo simulation for the dynamical SABR-HW model is presented. Numerical experiments for validation and calibration are discussed throughout the paper.

\section{The Dynamical SABR-HW Model}

This section describes the construction of the dynamical Stochastic Alpha Beta Rho Hull-White (SABR-HW) equity-interest rate model. We assume efficient markets and the existence of an equivalent martingale measure (EMM) $Q$ when appropriate numeraires need to be chosen.

\subsection{Model Definition}

We define the dynamical SABR-HW model in a similar fashion as Rebonato's SABRLMM model [26] for forward rates. The full-scale dynamical SABR-HW model for equity-interest rate products, under the Q-measure associated with $B(t)$, a money-saving 
account, is given by:

$$
\begin{aligned}
d S(t) / S(t) & =r(t) d t+\Sigma(t) S^{\beta-1}(t) d W_{x}(t), \quad S(0)>0, \\
d r(t) & =\lambda(\theta(t)-r(t)) d t+\eta d W_{r}(t), \quad r(0)>0, \\
\Sigma(t) & =g(t) \cdot k(t), \\
d k(t) & =h(t) k(t) d W_{\Sigma}(t), \quad k(0)=1, \\
d W_{x}(t) d W_{\sigma}(t) & =\rho_{x, \sigma} d t, \quad-1 \leq \rho_{x, \sigma} \leq 1, \\
d W_{x}(t) d W_{r}(t) & =\rho_{x, r} d t, \quad-1 \leq \rho_{x, r} \leq 1
\end{aligned}
$$

with constant parameters $\beta, \lambda, \eta, \rho_{x, r}$ and $\rho_{x, \sigma}$, and appropriately chosen time-dependent functions $\theta(t), g(t)$ and $h(t)$. For simplicity, we assume here that the interest rates are independent of the stochastic volatility, $d W_{r}(t) d W_{\sigma}(t)=0$. The parameters will be discussed in the subsections to follow, and details of the functional form of $g(t)$ and $h(t)$ are given in Section 4.2.

Remark. For some additional insight in the functions $g(t)$ and $h(t)$, we derive the dynamics of the time-dependent volatility $\Sigma(t)$, by applying Itô's lemma,

$$
\begin{aligned}
d \Sigma(t) \equiv d(g(t) k(t)) & =k(t) d g(t)+g(t) d k(t)+d k(t) d g(t) \\
& =\left(\frac{1}{g(t)} \frac{d g(t)}{d t}\right) \Sigma(t) d t+h(t) \Sigma(t) d W_{\Sigma}(t) \\
& =\frac{d \log g(t)}{d t} \Sigma(t) d t+h(t) \Sigma(t) d W_{\Sigma}(t) .
\end{aligned}
$$

Overall this implies that the dynamics for the volatility can be defined as:

$$
d \Sigma(t)=\hat{g}(t) \Sigma(t) d t+h(t) \Sigma(t) d W_{\Sigma}(t),
$$

with

$$
\hat{g}(t)=\frac{d \log g(t)}{d t},
$$

which means that we deal with a lognormal process with time-dependent drift and volatility terms. It is obvious that the function $h(t)$ plays the role of volatility coefficient for this volatility process and the function $g(t)$, appearing in the drift term, shifts the volatility up and down deterministically.

\subsubsection{The Hull-White Model}

One of the building blocks of hybrid model (2.1) is the Hull-White, single-factor, no-arbitrage yield curve model in which the short-term interest rate, $r(t)$, is driven by an Ornstein-Uhlenbeck (OU) mean reverting process, with $\theta(t)>0, t \in \mathbb{R}^{+}$a timedependent drift term, to fit theoretical bond prices to the yield curve observed in the market. Parameter $\eta$ determines the overall level of volatility and the reversion rate parameter, $\lambda$, determines the relative volatilities.

Under the Hull-White model the dynamics of the zero-coupon bond, paying $€ 1$ at maturity $T$, are given by:

$$
\frac{d P(t, T)}{P(t, T)}=r(t) d t+\frac{\eta}{\lambda}\left(\mathrm{e}^{-\lambda(T-t)}-1\right) d W_{r}(t) .
$$

Since the Hull-White model belongs to the class of affine diffusion processes, the solution of (2.2) is known analytically and reads:

$$
P(t, T)=\exp \left(A(t, T)+B_{r}(t, T) r(t)\right),
$$

with

$$
\begin{aligned}
B_{r}(t, T) & =\frac{1}{\lambda}\left(\mathrm{e}^{-\lambda(T-t)}-1\right), \\
A(t, T) & =\exp \left(\log \left(\frac{P(0, T)}{P(0, t)}\right)-B_{r}(t, T) f(0, t)-\frac{\eta^{2}}{4 \lambda}\left(1-\mathrm{e}^{-2 \lambda t}\right)\left(B_{r}(t, T)\right)^{2}\right),
\end{aligned}
$$


where $f(0, t):=-\partial P(0, t) / \partial t$ with $P(0, t)$ the market discount factor for maturity $t$.

By the Radon-Nikodým derivative [14],

$$
\frac{d \mathbb{Q}^{T}}{d \mathbb{Q}}=\frac{P(t, T)}{P(0, T) B(t)},
$$

we find the following change of measure: $d W_{r}^{T}(t)=d W_{r}(t)-\eta B_{r}(t, T) d t$.

Short rate, $r(t)$, under the $T$-forward measure is governed by the following dynamics:

$$
d r(t)=\left(\lambda(\theta(t)-r(t))+\eta^{2} B_{r}(t, T)\right) d t+\eta d W_{r}^{T}(t),
$$

which can be written as:

$$
d r(t)=\lambda(\hat{\theta}(t)-r(t)) d t+\eta d W_{r}^{T}(t),
$$

with $\hat{\theta}(t)=\theta(t)+\frac{\eta^{2}}{\lambda} B_{r}(t, T)$, and $B_{r}(t, T)$ in (2.4). Since the process under the $T$ forward measure in (2.7) is of 'Hull-White form', it is normally distributed [6] with expectation and variance given by:

$$
\begin{aligned}
\mathbb{E}^{T}(r(t)) & =r_{0} \mathrm{e}^{-\lambda t}+\lambda \int_{0}^{t} \hat{\theta}(s) \mathrm{e}^{-\lambda(t-s)} d s, \\
\operatorname{Var}^{T}(r(t)) & =\frac{\eta^{2}}{2 \lambda}\left(1-\mathrm{e}^{-2 \lambda t}\right) .
\end{aligned}
$$

A disadvantage of the Hull-White model is that it may give rise to negative interest rates. The negative interest rate, however, may be present in the real market ${ }^{1}$. An alternative to the Hull-White model is the Cox-Ingersoll-Ross (CIR) model. A hybrid SABR-CIR model is, however, not tractable if there is a non-zero correlation between the interest rate and the SABR equity process. The choice between a CIR or Hull-White model within the hybrid process is a trade-off between non-zero correlation and nonnegative rates.

For hybrid structured products, a non-zero correlation is a crucial feature that should be incorporated within a model (see Grzelak \& Oosterlee [12] for analysis and further arguments), whereas the appearance of negative interest rates in a Hull-White process is an inherent feature of the model and is known by practitioners for quite some time [6]. There are practical fixes to this problem, e.g. choosing parameters which give rise to lower probabilities for negative rates. We therefore prefer the HW process over the CIR process as part of our interest rate-equity hybrid model.

\subsubsection{Constant Parameter SABR-HW model}

The second building block of Model (2.1) is the Stochastic Alpha Beta Rho (SABR) stochastic volatility model by Hagan [18].

The SABR SDE system with constant parameters was originally defined under the $T$-forward measure, as:

$$
\begin{aligned}
d S(t) & =\sigma(t) S(t)^{\beta} d W_{x}^{T}(t), \\
d \sigma(t) & =\gamma \sigma(t) d W_{\sigma}^{T}(t),
\end{aligned}
$$

with $d W_{x}^{T}(t) d W_{\sigma}^{T}(t)=\rho_{x, \sigma} d t$.

One of the reasons why the original SABR model is not applied to equity derivatives is that a drift term is lacking. Risk-neutral equity price processes are defined with a drift term, and are assumed to be arbitrage-free under the risk-neutral measure associated with the money-savings account. For long-maturity equity options and equity interest rate hybrids, however, industrial practice is to treat the interest rate as a stochastic process as well. As shown below, when combining the Hull-White interest

\footnotetext{
${ }^{1}$ http://en.wikipedia.org/wiki/Interest_rate
} 
rate model with the SABR equity model the drift term appears naturally in the SABR equity dynamics under the risk neutral Q-measure:

$$
\begin{aligned}
& d S(t) / S(t)=r(t) d t+\sigma(t) S^{\beta-1}(t) d W_{x}(t), \quad S(0)>0, \\
& d \sigma(t)=\quad \gamma \sigma(t) d W_{\sigma}(t), \quad \sigma(0)>0, \\
& d r(t)=\lambda(\theta(t)-r(t)) d t+\eta d W_{r}(t), \quad r(0)>0,
\end{aligned}
$$

with constant model parameters $0<\beta<1, \gamma>0, \lambda>0$ and $\eta>0$. As in System (2.1), we assume non-zero correlations $d W_{x}(t) d W_{\sigma}(t)=\rho_{x, \sigma} d t, d W_{x}(t) d W_{r}(t)=\rho_{x, r} d t$ and $d W_{r}(t) d W_{\sigma}(t)=0$.

Since the interest rate diffusion coefficient in (2.11) is not explicitly dependent on the interest rate, it is convenient to move from the spot measure, generated by the money-savings account, $B(t)$, to the forward measure for which the numéraire is the zero-coupon bond, $P(t, T)$ :

$$
F(t):=\frac{S(t)}{P(t, T)}
$$

(details of $P(t, T)$ are given in (2.3))

By Itô's lemma the dynamics of forward price, $F(t)$, in (2.12) are given by:

$$
\begin{aligned}
d F(t) / F(t)= & \left(\eta^{2} B_{r}^{2}(t, T)-\rho_{x, r} \eta B_{r}(t, T) \sigma(t) S^{\beta-1}(t)\right) d t \\
& +\sigma(t) S^{\beta-1}(t) d W_{x}(t)-\eta B_{r}(t, T) d W_{r}^{T}(t),
\end{aligned}
$$

combined with the volatility process for $\sigma(t)$ in System (2.11). Since the forward, $F(t)$, is a martingale under the $T$-forward measure, the forward dynamics should not contain a drift term. This implies that " $d t^{\prime \prime}$-terms will not appear in the (reformulated) dynamics of $d F(t)$, i.e.:

$$
\begin{aligned}
d F(t) & =\sigma(t) F^{\beta}(t)\left(P^{\beta-1}(t, T) d W_{x}^{T}(t)-\frac{\eta B_{r}(t, T) F(t)}{\sigma(t) F^{\beta}(t)} d W_{r}^{T}(t)\right), \\
d \sigma(t) & =\gamma \sigma(t) d W_{\sigma}^{T}(t) .
\end{aligned}
$$

We assume that the interest rate is independent of the volatility process, so that a change of measure won't affect the dynamics of the variance process, $\sigma(t)$.

By factorization, Model (2.13) can be expressed as:

$$
\begin{aligned}
d F(t) & = & \sigma(t) v(t) F^{\beta}(t) d W_{F}^{T}(t), \\
d \sigma(t) & = & \gamma \sigma(t) d W_{\sigma}^{T}(t),
\end{aligned}
$$

with

$$
v^{2}(t):=P^{2(\beta-1)}(t, T)+\left(\frac{\eta B_{r}(t, T)}{\sigma(t) F^{\beta-1}(t)}\right)^{2}-2 \rho_{x, r} \frac{\eta B_{r}(t, T) P^{\beta-1}(t, T)}{\sigma(t) F^{\beta-1}(t)} .
$$

Now, the instantaneous correlation coefficient, $\rho_{F, \sigma}$, must be determined, which, by definition, is defined as:

$$
\rho_{F, \sigma}=\frac{\operatorname{Cov}(d F(t), d \sigma(t))}{\sqrt{\mathbb{V} \operatorname{ar}(d F(t)) \mathbb{V a r}(d \sigma(t))}}=\rho_{x, \sigma} \Psi(t, \sigma(t), F(t), P(t, T)),
$$

with $^{2}$

$$
\Psi(t, \sigma(t), F(t), P(t, T)):=\frac{F^{\beta} P^{\beta-1} \sigma}{\sqrt{\sigma^{2} F^{2 \beta} P^{2(\beta-1)}+\eta^{2} B_{r}^{2} F^{2}-\rho_{x, r} \sigma F^{\beta+1} P^{\beta-1} \eta B_{r}}} .
$$

Model (2.14) with (2.15) and (2.16) is not in the well-known plain SABR model form, because the local volatility is not only expressed by $\sigma(t) F^{\beta}(t)$ but contains additional terms, like $v(t)$. Moreover, the instantaneous correlation between forward and volatility processes, $\rho_{F, \sigma}$, is a state-dependent function of time. In order to make use of Hagan's asymptotic formulas [18] for the plain SABR model in the current setting, we propose a projection formula in the next section.

\footnotetext{
${ }^{2}$ To simplify notation we suppress the arguments $t$ and $T$ here.
} 


\section{Projection Formula for the Constant Parameter SABR- HW Model}

In this section we describe the model approximations that bring the SABR-HW model in the desired SABR model form. The approximations enable us to carry out efficient calibration based on the analytic implied volatility formulas for the SABR model.

\subsection{Projection Step for the Constant Parameter SABR-HW Model}

In order to present Model (2.14) in SABR form, we need to approximate the additional terms from the local volatility for the forward process, $F(t)$, and simplify the associated correlation structure. In a plain SABR model the volatility process, $\sigma(t)$, is lognormal, which suggests that a projection of the volatility term $\sigma(t) v(t)$ in (2.14) on a lognormal distribution may give the desired SABR form, which is:

$$
\begin{array}{rrr}
d F(t)= & \hat{\sigma}(t) F^{\beta}(t) d W_{F}^{T}(t), & F(0)>0, \\
d \hat{\sigma}(t)= & \hat{\gamma} \hat{\sigma}(t) d W_{\sigma}^{T}(t), & \hat{\sigma}(0)>0,
\end{array}
$$

with constant parameters $\hat{\sigma}(0)$ and $\hat{\gamma}$, and constant correlation $\hat{\rho}_{F, \sigma}$.

The term $v(t)$ in (2.15) depends on forward $F^{\beta-1}(t)$, volatility $\sigma(t)$ and on zerocoupon bond $P(t, T)$. With a function $v(t)$ which is independent of these state variables the expression simplifies. This can be achieved by freezing the forward and variance, $F(t)$ and $\sigma(t)$, at their initial values, i.e.: $F(t) \approx F(0), \sigma(t) \approx \sigma(0)$, respectively, and by projecting $P(t, T)$, on its expectation, i.e.: $P(t, T) \approx \mathbb{E}^{T}\left[P(t, T) \mid \mathcal{F}_{t}\right]=: \xi(t)$.

By this, function $v^{2}(t)$ is approximated by:

$$
v^{2}(t) \approx \xi^{2(\beta-1)}(t)+\left(\frac{\eta B_{r}(t, T)}{\sigma(0) F^{\beta-1}(0)}\right)^{2}-2 \rho_{x, r} \frac{\eta B_{r}(t, T) \xi^{\beta-1}(t)}{\sigma(0) F^{\beta-1}(0)} .
$$

With help of the well-known formulas (2.2) and (2.7), we obtain the following closedform solution for $\xi(t)$ :

$$
\xi(t)=\exp \left(A(t, T)+B_{r}(t, T) \mathbb{E}^{T}[r(t)]+\frac{1}{2} B_{r}^{2}(t, T) \operatorname{Var}^{T}(r(t))\right),
$$

with $\mathbb{E}^{T}[r(t)]$ and $\operatorname{Var}^{T}(r(t))$ given by (2.8) and (2.9), respectively, and $A(t, T)$ defined in (2.5).

Note that $F(t)$ and $\sigma(t)$ are both martingales, due to (2.14), which implies that the values of their expectations oscillate around their initial values, $F^{\beta-1}(0)$ and $\sigma(0)$. Function $v(t)$ has become deterministic by the approximations made.

We then determine the dynamics for the linearized volatility structure, $\bar{\sigma}(t):=\sigma(t) v(t)$. By applying the Itô product rule we find

$$
d \bar{\sigma}(t) / \bar{\sigma}(t)=v^{\prime}(t) d t+\gamma v(t) d W_{\sigma}^{T}(t) .
$$

The $\bar{\sigma}(t)$-dynamics are thus governed by a state-dependent drift term. They are therefore not yet in standard SABR volatility form, which does not contain any drift term.

However, since $v(t)$ is approximated by a deterministic time-dependent function, the process $\bar{\sigma}(t):=v(t) \sigma(t)$ remains lognormal. The idea is now to determine the first two moments of process $\bar{\sigma}(t)$ and to project them onto the moments of the SABR volatility process in (3.1), defined as $d \hat{\sigma}(t)=\hat{\gamma} \hat{\sigma}(t) d W_{\sigma}^{T}(t), \hat{\sigma}(0)>0$, with parameters $\hat{\gamma}$ and $\hat{\sigma}(0)$.

The expectation and variance of process $\hat{\sigma}(t)$ in (3.1) are given by:

$$
\mathbb{E}^{T}[\hat{\sigma}(t)]=\hat{\sigma}(0), \quad \operatorname{Var}^{T}(\hat{\sigma}(t))=\hat{\sigma}^{2}(0)\left(\mathrm{e}^{\hat{\gamma}^{2} t}-1\right) .
$$

On the other hand, the expectation and the variance of $\bar{\sigma}(t)=v(t) \sigma(t)$ are given by:

$$
\mathbb{E}^{T}[\bar{\sigma}(t)]=v(t) \sigma(0), \quad \operatorname{Var}^{T}(\bar{\sigma}(t))=v^{2}(t) \sigma^{2}(0)\left(\mathrm{e}^{\gamma^{2} t}-1\right) .
$$


The main objective is to find the effective parameters $\hat{\sigma}(0)$ and $\hat{\gamma}$, so that the expectations and variances in (3.4) and (3.5) match.

By matching the expectations and variances, we arrive at the following optimization problem:

$$
\underset{\hat{\gamma}, \hat{\sigma}(0)}{\operatorname{argmin}}\left\{\begin{array}{r}
\int_{0}^{T}\left(\mathbb{E}^{T}[\hat{\sigma}(t)]-\mathbb{E}^{T}[\bar{\sigma}(t)]\right) d t, \\
\int_{0}^{T}\left(\operatorname{Var}^{T}(\hat{\sigma}(t))-\operatorname{Var}^{T}(\bar{\sigma}(t))\right) d t .
\end{array}\right.
$$

Typically the optimization problem in (3.6) is easy since the expectations and variances are analytic deterministic functions. In Result 3.1 a straightforward approach for parameter estimation is presented.

Result 3.1. A simple approximation for $\hat{\sigma}(0)$ is the averaged parameter estimate, given by:

$$
\int_{0}^{T} \mathbb{E}^{T}[\hat{\sigma}(t)] d t=\int_{0}^{T} \mathbb{E}^{T}[\bar{\sigma}(t)] d t \quad \Longrightarrow \quad \hat{\sigma}(0)=\frac{\sigma(0)}{T} \int_{0}^{T} v(s) d s .
$$

By matching the variances we obtain:

$$
\int_{0}^{T} \operatorname{Var}^{T}(\hat{\sigma}(t)) d t=\int_{0}^{T} \operatorname{Var}^{T}(\bar{\sigma}(t)) d t \quad \Longrightarrow \quad \hat{\gamma}=\frac{1}{T} \int_{0}^{T} \sqrt{\frac{1}{s} \log \left(\frac{\operatorname{Var}^{T}(\bar{\sigma}(s))}{\hat{\sigma}^{2}(0)}+1\right)} d s,
$$

with $\operatorname{Var}^{T}(\bar{\sigma}(t))$ given in (3.5).

In the search for the optimal parameters $\hat{\sigma}(0)$ and $\hat{\gamma}$, so that the constant SABR-HW model in (2.11) is connected to the SABR dynamics given by (3.1), the correlation $\rho_{F, \sigma}$ has not yet been included. This allows us to determine the effective correlation, $\hat{\rho}_{F, \sigma}$, independent of the other approximations. Since the equation for correlation $\rho_{F, \sigma}$ is involved and state-dependent, we seek for a simplification also here. By freezing the volatility, and forward to their initial values, and by projection of zero-coupon bonds on their expectations, the correlation in Equation (2.16) can be approximated by:

$$
\rho_{F, \sigma} \approx \rho_{x, \sigma} \Psi\left(t, \sigma(0), F(0), \mathbb{E}^{T}\left[P(t, T) \mid \mathcal{F}_{t}\right]=: \rho_{x, \sigma} \psi(t) .\right.
$$

In order to use Hagan's implied volatility SABR formula, the correlation must be constant, so we need to determine an averaged correlation, defined as:

$$
\hat{\rho}_{F, \sigma}=\frac{\rho_{x, \sigma}}{T} \int_{0}^{T} \psi(s) d s,
$$

with $\psi(t)$ given by (3.9).

The estimates obtained for $\hat{\gamma}, \hat{\sigma}(0)$ and $\hat{\rho}_{F, \sigma}$ allow us to use the Hagan implied volatility formula for the plain SABR model, as a first approximation in the calibration procedure.

Remark. Our approximations in (3.7) and (3.8) perform well for not-too long maturity times, like $T \leq 10 y$. In the case of larger maturity times, $(T \geq 10 y)$, we prefer to solve Problem (3.6) by an optimization routine, for example, the Nelder-Mead Simplex algorithm. Furthermore, the weighted Monte Carlo method, to be discussed in Section 4, will be used to improve the calibration in those cases.

In the next subsection we check the accuracy of the approximations developed for a few parameter sets. The SABR model in (3.1) with the parameters $\hat{\gamma}, \hat{\sigma}(0)$ and $\hat{\rho}_{F, \sigma}$ will be called the $S A B R-H W 1$ model here.

\subsection{Numerical Validation of the SABR-HW Projection Method}

We check the performance of our approximation model, SABR-HW1, in comparison with the constant parameter SABR-HW model. 
The numerical experiment is set up as follows. We first prescribe a set of parameters for the constant parameter SABR-HW model in (2.11) for which, by means of an Euler-based Monte Carlo scheme, the European option prices are simulated. Secondly, we compute the effective parameters $\hat{\gamma}, \hat{\sigma}(0)$ and $\hat{\rho}_{F, \sigma}$ by solving (3.6) and calculating (3.10). These parameters are inserted in the plain SABR model (3.1). For the resulting SABR-HW1 model we then calculate the corresponding implied volatilities by Hagan's asymptotic formula. We compare these results and, in addition, we determine the error in the case that $\gamma, \sigma(0)$ and $\rho_{x, \sigma}$ were used, instead of $\hat{\gamma}, \hat{\sigma}(0), \hat{\rho}_{x, \sigma}$.

The simulations have been performed with 100.000 paths and 20T steps. The initial stock price is set to $S(0)=0.8$, and the zero-coupon bonds, $P(0, T)$, have been generated by the Hull-White model with constant long-term mean, $\theta=0.03$. We also define the strikes, as in [24], with expiry times given by $T \in\{1,5,10,15\}$ years; the strikes are computed by the formula:

$$
\begin{aligned}
K_{n}(T) & =F(0) \exp \left(0.1 \delta_{n} \sqrt{T}\right), \quad \text { with } \\
\delta_{n} & =\{-1.5,-1.0,-0.5,0,0.5,1.0,1.5\},
\end{aligned}
$$

and $F(0)$ is as in (2.12). This formula for the strikes is convenient, since for $n=4$, the strikes $K_{4}(\cdot)$ are equal to the forward FX rates.

\begin{tabular}{c|c|c|c|c|c|c|c}
\hline par. & $\beta$ & $\gamma$ & $\lambda$ & $\eta$ & $\sigma(0)$ & $\rho_{x, \sigma}$ & $\rho_{x, r}$ \\
\hline set 1 & $30 \%$ & $30 \%$ & $20 \%$ & $1 \%$ & $20 \%$ & $-30 \%$ & $20 \%$ \\
set 2 & $50 \%$ & $40 \%$ & $1 \%$ & $0.5 \%$ & $20 \%$ & $-10 \%$ & $40 \%$ \\
set 3 & $40 \%$ & $10 \%$ & $60 \%$ & $0.1 \%$ & $30 \%$ & $-30 \%$ & $-30 \%$ \\
\hline
\end{tabular}

Table 3.1: Sets of parameters used in the simulations.

In Table 3.1 we present three different sets of parameters. For those sets we determine the estimators $\hat{\gamma}, \hat{\sigma}(0)$ and $\hat{\rho}_{F, \sigma}$. They are tabulated in Table 3.2.

\begin{tabular}{c|c||c|c|c|c}
\hline & estimators & $\mathrm{T}=1 \mathrm{y}$ & $\mathrm{T}=5 \mathrm{y}$ & $\mathrm{T}=10 \mathrm{y}$ & $\mathrm{T}=15 \mathrm{y}$ \\
\hline \multirow{2}{*}{ set 1 } & $\hat{\gamma}$ & $29.76 \%$ & $29.02 \%$ & $28.34 \%$ & $27.85 \%$ \\
& $\hat{\sigma}(0)$ & $20.30 \%$ & $21.51 \%$ & $23.01 \%$ & $24.56 \%$ \\
& $\hat{\rho}_{F, \sigma}$ & $-30.08 \%$ & $-30.25 \%$ & $-30.30 \%$ & $-30.29 \%$ \\
\hline \multirow{2}{*}{ set 2 } & $\hat{\gamma}$ & $39.75 \%$ & $38.93 \%$ & $38.16 \%$ & $37.50 \%$ \\
& $\hat{\sigma}(0)$ & $20.24 \%$ & $21.28 \%$ & $22.73 \%$ & $24.52 \%$ \\
& $\hat{\rho}_{F, \sigma}$ & $-10.00 \%$ & $-10.01 \%$ & $-9.97 \%$ & $-9.90 \%$ \\
\hline \multirow{2}{*}{ set 3 } & $\hat{\gamma}$ & $9.97 \%$ & $9.78 \%$ & $9.56 \%$ & $9.35 \%$ \\
& $\hat{\sigma}(0)$ & $30.26 \%$ & $31.36 \%$ & $32.83 \%$ & $34.39 \%$ \\
& $\hat{\rho}_{F, \sigma}$ & $-30.01 \%$ & $-30.02 \%$ & $-30.02 \%$ & $-30.02 \%$ \\
\hline
\end{tabular}

Table 3.2: Effective constant parameters $\hat{\gamma}, \hat{\sigma}(0)$ and $\hat{\rho}_{F, \sigma}$, defined in (3.6), and determined by solving the non-linear least squares problem (by Matlab function lsqcurvefit).

We measure the maximum absolute difference in the implied volatilities for Model (3.1) with the estimates in (3.7), (3.8), and the constant parameter SABR-HW model (2.11).

Two errors are defined: error 1 is the error when the naive approach is used, i.e. $\hat{\gamma}=\gamma$ and $\hat{\sigma}(0)=\sigma(0)$; error 2 corresponds to the bias obtained using the adjusted parameters $\hat{\gamma}$ and $\hat{\sigma}(0)$ (the SABR-HW1 model). Table 3.3 presents these results.

Our approach for $\hat{\gamma}, \hat{\sigma}(0)$ and $\hat{\rho}_{F, \sigma}$ provides a significantly better fit to the constant parameter SABR-HW model than the model with the naively chosen parameters. For the maturity times of 1,5, and 15 years, Figure 3.1 presents the corresponding implied volatilities. The performance of the SABR-HW1 approximation is most accurate when the volatility for the short rate, determined by $\eta$, is not too large, i.e. $\eta<1.5 \%$. Fortunately, this is very often the case in the calibration of the Hull-White model to market 


\begin{tabular}{c|c|c||c|c||c|c||c|c}
\hline & \multicolumn{2}{|c|}{$\mathrm{T}=1 \mathrm{y}$} & \multicolumn{2}{c||}{$\mathrm{T}=5 \mathrm{y}$} & \multicolumn{2}{c||}{$\mathrm{T}=10 \mathrm{y}$} & \multicolumn{2}{c}{$\mathrm{T}=15 \mathrm{y}$} \\
\cline { 2 - 9 } & error 1 & error 2 & error 1 & error 2 & error 1 & error 2 & error 1 & error 2 \\
\hline set 1 & $0.36 \%$ & $0.02 \%$ & $1.63 \%$ & $0.19 \%$ & $2.93 \%$ & $0.43 \%$ & $3.94 \%$ & $0.61 \%$ \\
set 2 & $0.27 \%$ & $0.01 \%$ & $1.38 \%$ & $0.08 \%$ & $2.65 \%$ & $0.32 \%$ & $3.74 \%$ & $0.51 \%$ \\
set 3 & $0.31 \%$ & $0.03 \%$ & $1.63 \%$ & $0.04 \%$ & $3.12 \%$ & $0.07 \%$ & $4.31 \%$ & $0.11 \%$ \\
\hline
\end{tabular}

Table 3.3: The absolute maximum percentage difference between implied volatilities from two different models. Both errors relate to the constant parameter SABR-HW model and respective approximations.
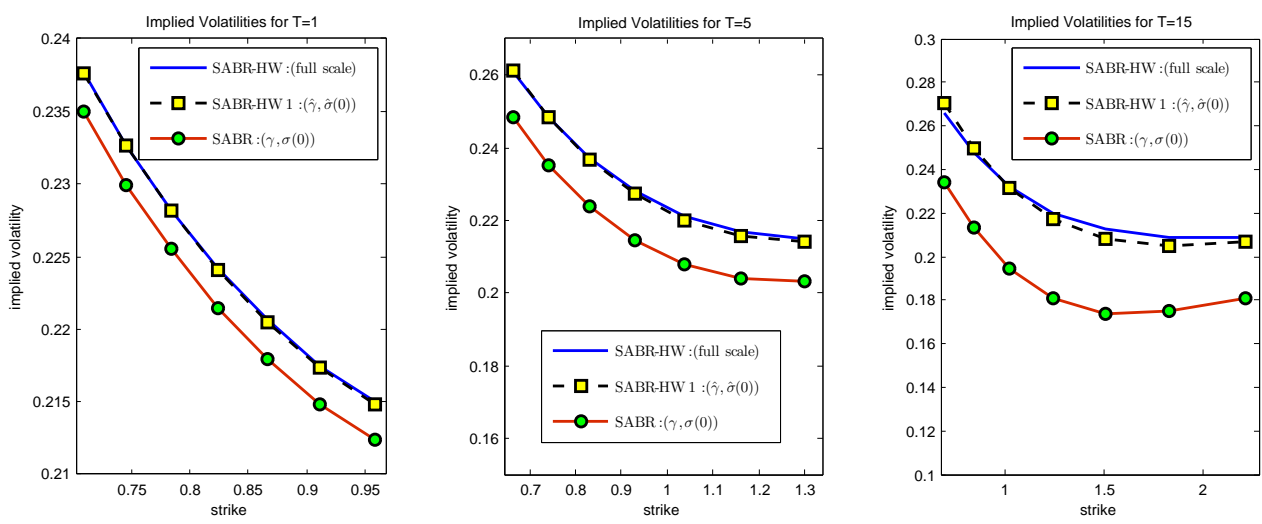

Figure 3.1: Comparison of implied Black-Scholes volatilities for European equity options and parameter set 2 in Table 3.1; For the SABR-HW model, Euler Monte Carlo was used with 100.000 paths and 20T intermediate steps.

data. In the experiments we have chosen parameter $\beta \leq 50 \%$ (as in the case $\beta>50 \%$ an even better accuracy is expected, because then the model behavior is closer to that of a lognormal model [10]). We also see that correlation approximation, $\hat{\rho}_{F, \sigma}$, is close to the initial correlation $\rho_{F, \sigma}$. This is because the function $\Psi(t, \sigma(t), F(t), P(t, T))$ in (2.17) converges to 1 as $t \rightarrow T$, implying that $\rho_{F, \sigma} \rightarrow \hat{\rho}_{F, \sigma}$.

\section{The Calibration Procedure}

We present a calibration procedure for the SABR-HW model in three stages, and start by applying the inverse projection formulas from the previous section to calibrate the constant parameter SABR-HW model for every single maturity. In the second stage, we determine the parameters of the time-dependent functions in the dynamical SABR model in order to produce coherent model dynamics across the different maturities. In the final stage, the calibration is refined by means of a weighted Monte Carlo simulation. These stages are discussed in subsequent subsections.

\subsection{Stage I: Parameter Projection for the SABR-HW Model}

In the calibration of the SABR-HW model, the Hull-White part, which is connected to the function $\theta(t)$, is calibrated to the yield curve, whereas the parameters $\lambda$ and $\eta$ are calibrated to swaption prices separately. This is well-known and we refer to Brigo \& Mercurio [6] for further information on this topic. The asset-interest rate correlation will be prescribed a priori based on historical data.

After the calibration of the HW model, we consider the determination of the parameters of the stochastic volatility SABR part.

One of the consequences of the projection of the constant parameter SABR-HW model onto a plain SABR model is the rapid calibration by means of Hagan's for- 
mula [30]. The projection formula, described in Section 3, can also be inverted numerically to retain the constant parameter SABR-HW parameters, $\sigma(0), \gamma, \rho_{x, \sigma}, \rho_{x, r}, \lambda, \theta(t), \eta$, from those of a plain SABR model, $\hat{\sigma}(0), \hat{\gamma}, \hat{\rho}_{F, \sigma}$. Since two parameters, $\beta$ and $\rho_{x, \sigma}$, control the skewness of the implied volatility curve, one of them (parameter $\beta$ in our case) is fixed a-priori, as in Rebonato [28].

We briefly recall the calibration of the plain SABR model, in which for $\beta$ different values are prescribed, like $\beta \in\{0.25,0.5,0.75,1\}$, see for example [30], [28]. By numerical experiments we observe that different combinations of $\beta$ and $\rho$ give rise to parameter fits of very similar quality. This is especially true for short maturity implied volatilities (see Figure 4.1). The specific $\beta$ which gives the best fit for both short and long maturities will be determined in the second calibration stage.

Parameter $\hat{\sigma}(0)$ is determined with the help of the at-the money (ATM) implied volatility. West [30] showed that when the forward in the plain SABR model is equal to the strike price, $F=K$, the ATM implied volatility in Hagan's formula simplifies to:

$$
\sigma_{A T M}=\frac{\hat{\sigma}(0)\left(1+\left(\frac{(1-\beta)^{2}}{24} \frac{\hat{\sigma}(0)^{2}}{F^{2-2 \beta}}+\frac{1}{4} \frac{\hat{\rho}_{F, \sigma} \beta \hat{\gamma} \hat{\sigma}(0)}{F^{1-\beta}}+\frac{2-3 \hat{\rho}_{F, \sigma}^{2}}{24} \hat{\gamma}^{2}\right) T\right)}{F^{1-\beta}} .
$$

This equation is inverted, as in [30], to calculate $\hat{\sigma}(0)$ as a root of the cubic equation:

$$
\frac{(1-\beta)^{2} T}{24 F^{2-2 \beta}} \hat{\sigma}(0)^{3}+\frac{\hat{\rho}_{F, \sigma} \beta \hat{\gamma} T}{4 F^{1-\beta}} \hat{\sigma}(0)^{2}+\left(1+\frac{2-3 \hat{\rho}_{F, \sigma}^{2}}{24} \hat{\gamma}^{2} T\right) \hat{\sigma}(0)-\sigma_{A T M} F^{1-\beta}=0 .
$$

For typical parameters the above cubic equation has only one real-valued root (and two imaginary roots), but it is in general possible to have three real-valued roots. In such cases, the smallest positive root should be chosen [30]. As a result of the cubic equation formulation, $\hat{\sigma}(0)$ is not a free variable anymore, but a function of the parameters $\hat{\rho}_{F, \sigma}$, $\hat{\gamma}$ and the market ATM implied volatility, $\sigma_{\mathrm{ATM}}$. Subsequently, the calibration only has to be performed over the parameters $\hat{\rho}_{F, \sigma}$ and $\hat{\gamma}$, which can be done very efficiently.

From Eq. (3.7), we know that $\hat{\sigma}(0)=\sigma(0) / T \int_{0}^{T} v(s) d s$, where

$$
v(t) \approx \sqrt{\xi^{2(\beta-1)}(t)+\left(\frac{\eta B_{r}(t, T)}{\sigma(0) F^{\beta-1}(0)}\right)^{2}-2 \rho_{x, r} \frac{\eta B_{r}(t, T) \xi^{\beta-1}(t)}{\sigma(0) F^{\beta-1}(0)}} .
$$

So, $\hat{\sigma}(0)$ itself is a function of $\sigma(0)$ (since the other parameters and functions in the equation for $v(t)$ have been determined in earlier steps). Applying a numerical rootfinding routine provides us with a value for $\sigma(0)$. Similarly, we can find the solution for $\gamma$ via Eqs. (3.8) and (3.5). With Formula (2.16) we can rewrite the correlation $\hat{\rho}_{F, \sigma}$ as a function of $\rho_{x, \sigma}$. The numerical inversion of this expression gives us parameter $\rho_{x, \sigma}$. After this, all parameters of the SABR-HW system, $\sigma(0), \gamma, \rho_{x, \sigma}, \rho_{x, r}, \lambda, \theta(t), \eta$, have been determined.

This stage of the calibration procedure is highly efficient as most of the evaluations are based on analytic expressions. The numerical root-finding procedure is used four times. The overall CPU time is less than a second.

We present an example of the calibration of the parameters $\hat{\rho}_{F, \sigma}$ and $\hat{\gamma}$ to the 5- and 15 -years DAX options with equally spaced strike values from $40 \%$ to $220 \%$ with $10 \%$ intervals (in total 19 strikes), from 27-09-2010, based on the procedure described. The calibration has been performed with 4 sets of parameters with different a priori chosen values for $\beta$. The whole procedure (for the four sets of parameters) takes approximately 0.3 seconds CPU time on a desktop computer. The resulting parameters and squared sum errors (SSE) are presented in Table 4.1. Different values of $\beta$ result in a qualitatively similar fit to the market implied volatilities. The fit of the SABR-HW model (based on Hagan's formula) to the market implied volatilities is presented in Figure 4.1.

The last three columns in Table 4.1, the columns $\sigma(0), \gamma$ and $\rho_{F, \sigma}$, present the constant parameter SABR-HW model parameters obtained from the inversion of the projection formulas described above. We see that constant parameters produce a very 
Table 4.1: Calibrated parameters for the SABR (parameters with hat) and SABR-HW (without hat) models, to 5 and 15 years DAX options.

\begin{tabular}{cccccccc}
\hline \hline$\beta$ & $\hat{\sigma}(0)$ & $\hat{\gamma}$ & $\hat{\rho}_{F, \sigma}$ & SSE & $\sigma(0)$ & $\gamma$ & $\rho_{F, \sigma}$ \\
\hline \hline \multicolumn{7}{c}{$\mathrm{T}=5$ years } \\
\hline 0.25 & 0.280 & 0.033 & -1 & $3.82 \times 10^{-4}$ & 0.274 & 0.034 & -0.964 \\
0.5 & 0.276 & 0.113 & -0.955 & $2.22 \times 10^{-6}$ & 0.269 & 0.117 & -0.922 \\
0.75 & 0.274 & 0.224 & -0.824 & $9.94 \times 10^{-6}$ & 0.267 & 0.234 & -0.800 \\
1 & 0.279 & 0.319 & -0.840 & $6.53 \times 10^{-5}$ & 0.272 & 0.333 & -0.815 \\
\hline \hline \multicolumn{7}{c}{$\mathrm{T}=15$ years } \\
\hline 0.25 & 0.402 & $10^{-7}$ & -1 & $1.16 \times 10^{-4}$ & 0.369 & $1.0134 \times 10^{-7}$ & -0.942 \\
0.5 & 0.356 & $10^{-7}$ & -1 & $1.75 \times 10^{-5}$ & 0.327 & $1.0134 \times 10^{-7}$ & -0.937 \\
0.75 & 0.328 & $10^{-7}$ & -1 & $1.73 \times 10^{-6}$ & 0.301 & $1.0134 \times 10^{-7}$ & -0.933 \\
1 & 0.319 & 0.033 & -0.758 & $3.89 \times 10^{-8}$ & 0.292 & 0.033 & -0.722 \\
\hline
\end{tabular}

SSE: squared sum errors
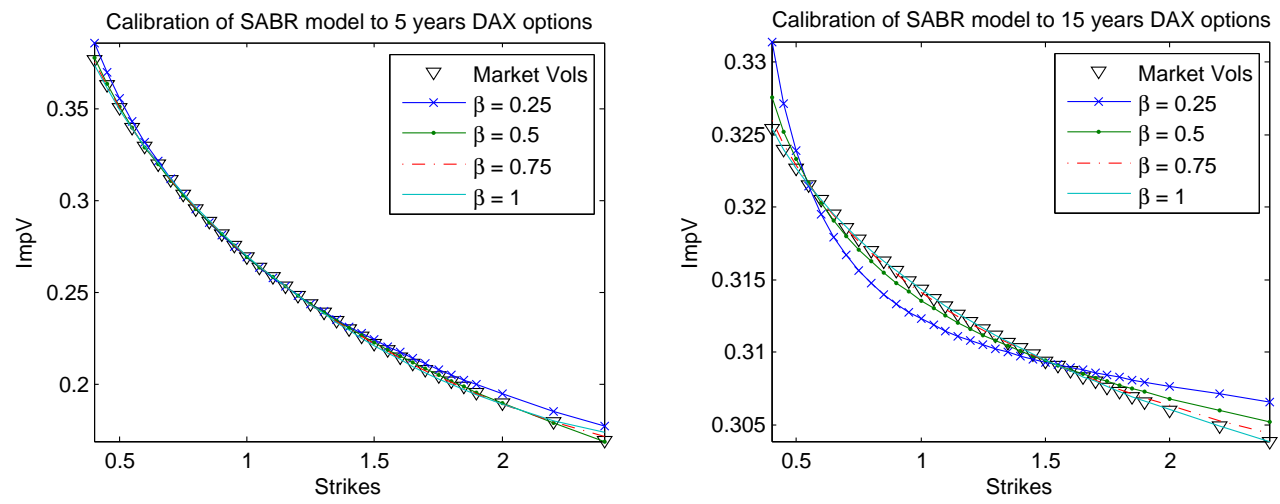

Figure 4.1: Calibration results for the SABR model with different $a$ priori chosen $\beta$ parameters to the implied volatilities of 5 and 15 years maturity. 
Table 4.2: Parameters $a_{1}, b_{1}, c_{1}$ and $d_{1}$ for calibrated function $h(\cdot)$ and $a_{2}, b_{2}, c_{2}$ and $d_{2}$ for function $g(\cdot)$ for DAX option implied volatilities on 27-09-2010.

\begin{tabular}{ccccc}
\hline Parameters & $a_{1}\left(a_{2}\right)$ & $b_{1}\left(b_{2}\right)$ & $c_{1}\left(c_{2}\right)$ & $d_{1}\left(d_{2}\right)$ \\
\hline$h(\cdot)$ & 0.5928 & -0.1943 & 1.1936 & 0.1080 \\
$g(\cdot)$ & 0.0949 & 0.0673 & 0.1297 & 0.0858 \\
\hline
\end{tabular}

good fit for individual maturity times, but the resulting parameters differ for different maturities.

\subsection{Stage II: Calibration of the Dynamical SABR-HW Model}

Calibration of the constant parameter SABR-HW model results in a series of independent implied volatility smiles across several maturities, which do not show coherent dynamics over a longer time period. We therefore describe here the calibration of the time-dependent functions in the dynamical SABR-HW system (2.1), and start with the parameters $\beta$ and $\rho_{x, \sigma}$ for this dynamical SDE system. The value for $\beta$ which fits optimally for all maturities (e.g. the optimal value from Table 4.1) is chosen, and simultaneously we average the calibrated correlation parameters, $\rho_{x, \sigma}$, for the corresponding $\beta$-value, over the different maturity times.

Then, the time-dependent function $h(t)$ in System (2.1) is parametrized in the form proposed by Rebonato [26]:

$$
h(t)=\left(a_{1}+b_{1} t\right) \exp \left(-c_{1} t\right)+d_{1} .
$$

The parameters $a_{1}, b_{1}, c_{1}$ and $d_{1}$ are determined, as in [27], by solving the following system of equations for all maturities $T_{i}$ included in the calibration instruments:

$$
\gamma^{T_{i}}-\frac{1}{\sigma^{T_{i}}(0) T_{i}} \sqrt{2 \int_{0}^{T} g(t)^{2} \hat{h}(t)^{2} t d t}=0 .
$$

Here superscript $T_{i}$ denotes the maturity for which the parameter is determined, and $\hat{h}(t)$ denotes the mean value of $h(\cdot)$ up to time $t$, i.e.

$$
\hat{h}(t)=\sqrt{\frac{1}{t} \int_{0}^{t}(h(s))^{2} d s .}
$$

Eq. (4.2) can be best dealt with by a numerical root finding technique.

For the time-dependent function $g(t)$, a common parametrization is:

$$
g(t)=\left(a_{2}+b_{2} t\right) \exp \left(-c_{2} t\right)+d_{2},
$$

which can also be found in Brigo \& Mercurio [6] or Rebonato [25]. We obtain $a_{2}, b_{2}, c_{2}$ and $d_{2}$ by minimization of the sum of squared errors:

$$
\min _{a_{2}, b_{2}, c_{2}, d_{2}} \sum_{i=1}^{M}\left[\sigma^{T_{i}}(0)-\hat{g}(t)\right]^{2}, \hat{g}(t)=\sqrt{\frac{1}{T_{i}} \int_{0}^{T_{i}}\left[\left(a_{2}+b_{2} t\right) \exp \left(-c_{2} t\right)+d_{2}\right]^{2} d t}
$$

with $M$ the number of option maturity times.

The time-dependent functions, $g(t)(4.3)$ and $h(t)(4.1)$, are then fitted for all maturity times to the parameters $\sigma(0)$ and $\gamma$ obtained from the constant parameter calibration. The resulting parameters are presented in Table 4.2. The functions are illustrated in Figure 4.2. We fix the parameter $d_{1}$ in function $h(t)$ to the value of the volatilityof-volatility parameter of the longest maturity to prevent it from attaining negative values.

Remark: The Hagan implied volatility function [18] is based on asymptotic expansions that have a limited range of applicability. The formula is not exact, for example, 


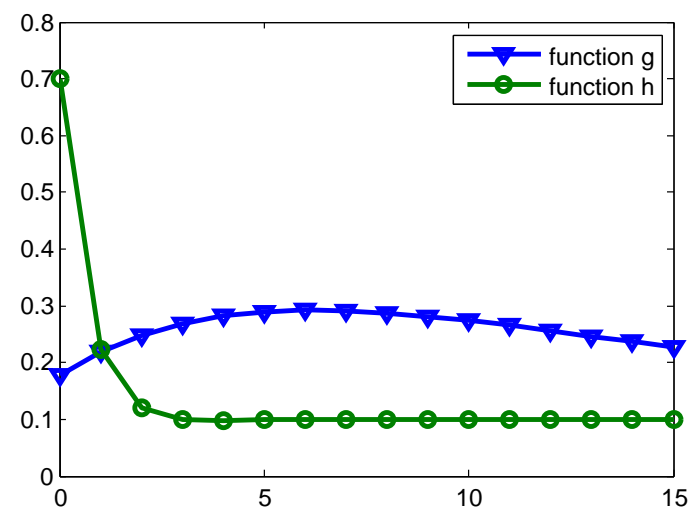

Figure 4.2: The calibrated $g(\cdot)$ and $h(\cdot)$ functions.

for deep out-of-the-money strikes, especially for strikes close to zero, and for long maturities. Thus, the model dynamics that are simulated by the Monte Carlo technique may not resemble the parameters determined during calibration. In the next section, we will propose a method to eliminate such approximation error induced calibration error.

\subsection{The Weighted Monte Carlo Technique}

We employ a non-parametric approach to further improve the SABR-HW model calibration. The general idea is to perturb the weights of the individual Monte Carlo paths so that calibration instruments, like options, forwards and bonds, resemble the corresponding market prices in a better way.

Most often, one deals with ordinary Monte Carlo methods, that are governed by the fact that the same weight (i.e. $1 / N$, with $N$ the total number of paths) is assigned to each sampled path. For a claim with a payoff, $\phi$, the derivative value, at $t=0$, is then determined as:

$$
V=\frac{1}{N} \sum_{i=1}^{N} \phi\left(\omega_{i}\right),
$$

where $\omega_{i}$ denotes the $i$-th Monte Carlo path.

In addition, weighted Monte Carlo methods have been developed by Avellaneda et al. $[3 ; 4 ; 5]$, in which different 'probability' weights, $p_{1}, p_{2}, \ldots, p_{N}$, are assigned to the individual Monte Carlo paths. The value of the claim then reads:

$$
V=\sum_{i=1}^{N} \phi\left(\omega_{i}\right) p_{i} .
$$

The weights are determined so that the model values of the calibration instruments match well with the market prices and these weights should be kept as close as possible to the uniform weights $\left(p_{i}=\frac{1}{N}\right)$.

We denote the market prices of $M$ calibration instruments by $C_{1}, \ldots, C_{M}$ and represent the present values of the derivative products of the $j$-th calibration instruments along path $\omega_{i}$ by $\phi_{i j}, j=1,2, \ldots, M$. The first index represents the Monte Carlo path number and the expression is short notation for $\phi_{i j} \equiv \phi_{j}\left(\omega_{i}\right)$.

The path weights, or probabilities $p=\left(p_{1}, p_{2}, \ldots, p_{N}\right)$, have to be determined, so that

$$
\sum_{i=1}^{N} p_{i} \phi_{i j}=C_{j}
$$


or so that the difference between the left- and right-hand side is minimized. A criterion (which is adopted here) to find these weights is the minimization of the relative entropy of a non-uniformly sampled probability with respect to a uniform distribution.

The concept of relative entropy is not new in computational finance. Buchen \& Kelly [9] proposed the use of the minimization of relative entropy to determine the Arrow-Debreu probability in a single-period model. This method was generalized to dynamical models by Avellaneda et al. [3; $4 ; 5]$.

Based on two sets of $N$ discrete probabilities, $p=\left(p_{1}, p_{2}, \ldots, p_{N}\right)$ and $q=\left(q_{1}, q_{2}, \ldots, q_{N}\right)$, the relative entropy of $p$, with respect to $q$, is defined as

$$
D(p \| q):=\sum_{i=1}^{N} p_{i} \log \left(\frac{p_{i}}{q_{i}}\right) .
$$

In the case of a Monte Carlo simulation, in which $q_{i}=1 / N, \forall i$, we have

$$
D(p \| q)=\sum_{i=1}^{N} p_{i} \log \left(p_{i}\right)-\sum_{i=1}^{N} p_{i} \log \left(\frac{1}{N}\right)=\sum_{i=1}^{N} p_{i} \log \left(p_{i}\right)+\log (N),
$$

where we used that $\sum_{i=1}^{N} p_{i}=1$. The objective is to minimize Eq. (4.5) under the linear constraints implied by Eq. (4.4). A true advantage of the relative entropy objective function lies in the fact that Eq. (4.5) is convex in all $p_{i}$-values ${ }^{3}$. It is well-known in optimization theory that the above minimization problem has a unique global minimum solution, if it exists [8], and that the Lagrange multiplier technique determines this solution in an efficient way.

Here we present results obtained by the WMC approach and refer for details to Avellaneda's original paper [5].

In probability and in information theory, the relative entropy, or Kullback-Leibler divergence, is a so-called non-symmetric measure of the difference between two probability distributions $p$ and $q$. The relative entropy measure is an indication for the difference between any two models. In our case, it quantifies the consistency, or inconsistency, between the calibrated true SABR-HW model and the SABR-HW1 model obtained from the first stages of the calibration. The relative entropy distance is defined as $D(p \| u)$ in Eq. (4.5), in which $u$ denotes a uniform probability of $N$ Monte Carlo samples. Since the term $\sum_{i=1}^{N} p_{i} \log p_{i}$ in Eq. (4.5) is negative (as $\left.p_{i} \leq 1\right), D(p \| u) \in[0, \log N]$. The minimum value, $D(p \| u)=0$, corresponds to $p_{i}=1 / N$, i.e. the calibrated vector $p$ equals the prior $u$. The maximum value, $D(p \| u)=\log N$, is realized when the probability is concentrated at a single path, i.e. $p_{i}=1$. Consider a probability distribution which is uniformly distributed on a subset of paths of size $N^{\alpha}$, with $0<\alpha<1$. Substitution of the corresponding probabilities, gives [5]:

$$
D(p \| u)=\log N+\log \left(\frac{1}{N^{\alpha}}\right)=(1-\alpha) \log N,
$$

using $\sum_{i=1}^{N^{\alpha}} N^{-\alpha}=1$.

The relative entropy distance can thus be connected to the effective number of paths, $N^{\alpha}$, supported by the prior probability measure. The effective number of paths can be obtained, as $\alpha=1-D(p \| u) / \log N$, with $D(p \| u) / \log N \in[0,1]$. If $D(p \| u) / \log N<<$ 1 the number of significant paths is close to $N$, whereas $D(p \| u) / \log N \approx 1$ is connected to a measure with 'thin support' [5]. Thin support implies that in the calibration a large number of paths is discarded, which is inefficient from a computational point of view.

Remark. It is possible that a solution to the minimum entropy problem does not exist, when the initial problem parameters result in prices of the calibration instruments

\footnotetext{
${ }^{3}$ It is straightforward to show that

$$
\frac{\partial D(p \| q)}{\partial p_{i}}=\log \left(p_{i}\right)+1, \quad \frac{\partial^{2} D(p \| q)}{\partial p_{i}^{2}}=\frac{1}{p_{i}}>0 .
$$
}



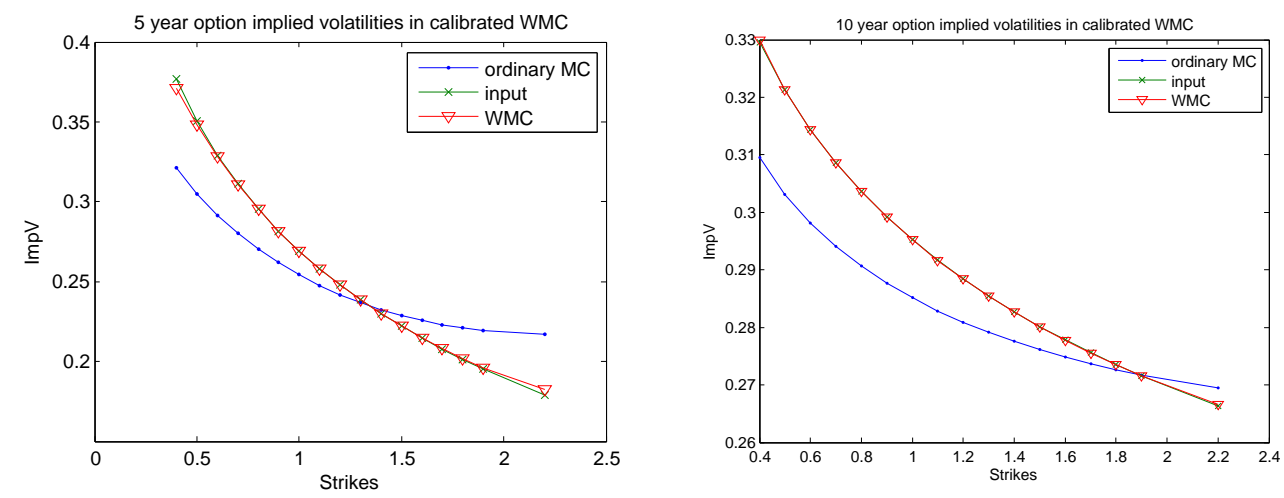

Figure 4.3: The 5 and 10 year option implied volatilities produced by WMC compared against the input market implied volatilities.
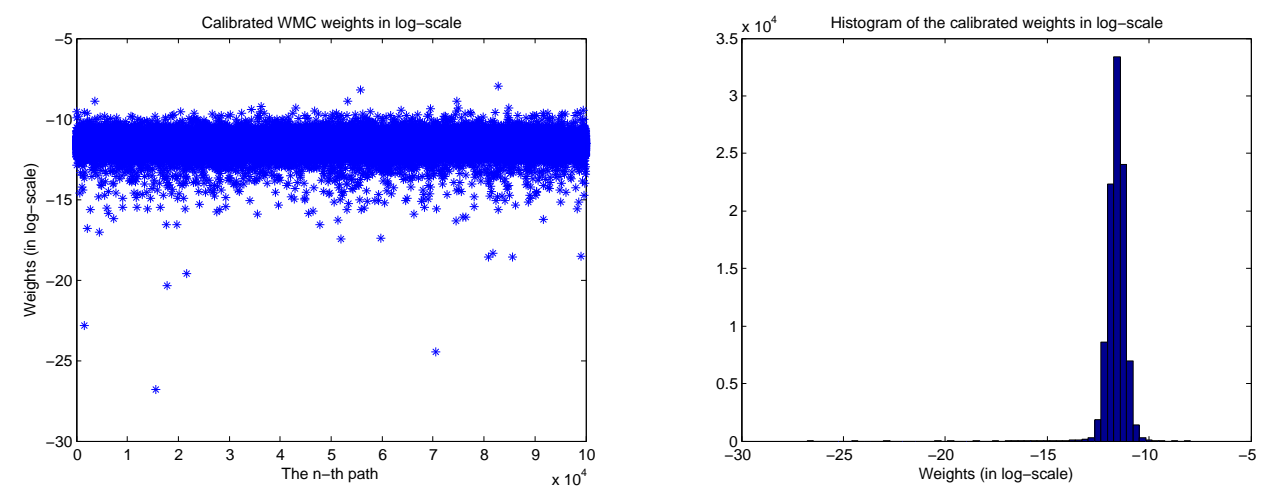

Figure 4.4: Left: a cloud plot of the $10^{5}$ weights (in log-scale) of the WMC paths obtained after the calibration. Right: histogram of the values of the calibrated weights in log-scale.

that are very different from the market prices. In such a case, the minimum entropy algorithm won't work but one may use a quadratic difference function,

$$
D^{Q}(p \| u)=\sum_{i=1}^{N}\left(p_{i}-\frac{1}{N}\right)^{2}
$$

instead of the relative entropy distance, which guarantees a solution, see [2].

\subsection{Stage III: Calibration by Weighted Monte Carlo Method}

Here, we use the DAX 1-, 5- and 10-years implied volatilities from 27-09-2010 with equally spaced strike values from $40 \%$ to $220 \%$ with $10 \%$ intervals. After the computation of the WMC weights for these financial derivatives, the weighted Monte Carlo method perfectly replicates the prices these calibration instruments (see Figure 4.3).

We plot the resulting WMC weights in log-scale at the left-hand graph of Figure 4.4. The weights seem to be randomly distributed around their mean value of $10^{-5}$. Certain paths are given a small weight, which means that these paths are effectively discarded. The histogram of the weights at the right-hand side of Figure 4.4 indicates that only a small fraction of the weights is in the left tail and most of them is distributed around the mean. The resulting effective number of paths, obtained by Eq. (4.6), is $9.3517 \times 10^{4}$, so that $6.48 \%$ of the paths are discarded. This is efficient from a computational point of view, given the excellent WMC calibration results.

Remark: The WMC calibration procedure is highly efficient, but it is non-parametric, and this may hamper its practical application. If either the model or the imodel parame- 
ters are not carefully chosen, too many paths will be discarded and the WMC efficiency would be lost. The WMC technique can however also be used as an a posteriori check of the quality of parameters obtained from calibration to Hagan's implied volatilities. If, after the first stage of calibration, the Monte Carlo weights are equally distributed and very close to $p_{i}=1 / N$, this may give confidence in the quality of the first stage of calibration.

\section{Pricing Options under the SABR-HW Model}

On the basis of the calibrated SABR-HW model, we are now ready to apply a (weighted) Monte Carlo simulation for the pricing and hedging of exotic derivatives. We present an advanced time stepping scheme for the Monte Carlo simulation, leading to a low-bias Monte Carlo simulation. This scheme is accurate also when only a few time steps are employed. It has also been used within the WMC part of the calibration procedure, described previously.

\subsection{Low-Bias Time Discrete Scheme}

Applications of the SABR-HW model include the pricing of long-maturity equity options, equity-linked structured notes, like cliquet options, and equity-linked hybrid derivatives. Structured products usually have a long time horizon and a complicated payoff. It is difficult to find analytic approximations for these product prices, and often one has to rely on Monte Carlo methods to obtain prices and hedge ratios (e.g. price sensitivities, the Greeks). If we apply an Euler discretization scheme to the SABR-HW system, the discrete bias has to be analyzed with care. For example, in Eq. (2.11) the drift term is stochastic and driven by two stochastic factors. In this case, an Euler approximation for the drift term,

$$
\int_{0}^{\Delta} r(s) S(s) d s \approx r(0) S(0) \Delta,
$$

is biased in general and a large number of time steps is required to reach an acceptable level of accuracy. An Euler scheme may therefore be inefficient for pricing long-term equity-linked structured products.

Here we adapt the low-bias MC scheme proposed for the SABR model in Chen [11] to discretize the SABR-HW model. The approach is to map the asset price process onto a square root process by a series of spatial and time transformations. In the Monte Carlo simulation we will draw samples from the analytic distribution function of the square root process (i.e. the non-central chi square distribution), as described in Section 5.2, and, in full detail, in [11].

The SABR model considered in Chen [11] was develoepd for a system without drift term. A stochastic interest rate can be incorporated by a technique described in Goldenberg [16] (pp. 28), which was introduced for a Constant Elasticity of Variance (CEV) process with drift term, $r S(t) d t$ :

$$
d S^{(r)}(t)=r S^{(r)}(t) d t+\sigma S^{(r)}(t)^{\beta} d W(t) .
$$

We use the superscript $(r)$ to distinguish the process with drift from the process without drift, which does not have a superscript. The distribution of the CEV process in (5.1) can be obtained from its sister without drift via a time change:

$$
S^{(r)}(t)=e^{r t} S(\tau(t)), \quad \tau(t)=\frac{1}{2 r(\beta-1)}\left(e^{2 r(\beta-1) t}-1\right) .
$$

The validity of this transformation can easily be explained as the limit $r \rightarrow 0$ recovers the original clock, i.e.,

$$
\lim _{r \rightarrow 0} \tau(t)=\lim _{r \rightarrow 0} \frac{1}{2 r(\beta-1)}\left(e^{2 r(\beta-1) t}-1\right)=t .
$$


This result is not restricted to the constant interest rate case. The time transformation in (5.2) also applies to stochastic interest rates [16].

In an SDE system with a stochastic interest rate, the time transformation is different for each path, due to the randomness of the rates:

$$
\tau(t, \omega)=\frac{\Delta}{2(\beta-1) \int_{0}^{\Delta} r(s) d s}\left(e^{2(\beta-1) \int_{0}^{\Delta} r(s) d s}-1\right),
$$

where $\omega \in \Omega$ denotes a random scenario. Expression (5.3) suggests that the pathwise time transformation, $\tau(t, \omega)$, can be determined without all details of the interest rate path, $\omega$, as long as we have knowledge of $\int_{0}^{\Delta} r(s) d s$ at each path.

Following the arguments by Andersen [1], we focus on the evolution of the system over a small time interval $[0, \Delta]$ and repeat the one-period $\Delta$-scheme to produce a complete time discrete path. Note that we consider the SDE system in the $\tau$-time scale, so that the time interval for the system equals $[0, \tau(\Delta, \omega)]$.

The CEV system in (5.2) is then simulated on a time scale $\tau(t, \omega)$, induced by a stochastic interest rate. Subsequently, the result is multiplied by an exponentially integrated interest rate:

$$
\begin{aligned}
& S(\tau(\Delta, \omega))=S(0)+\int_{0}^{\tau(\Delta, \omega)} \sigma(\tau(s, \omega)) S(\tau(s, \omega))^{\beta} d W_{x}(\tau(s, \omega)), \\
& S^{(r)}(\Delta)=\exp \left(\int_{0}^{\Delta} r(s) d s\right) S(\tau(\Delta, \omega)) .
\end{aligned}
$$

Although a transformed model based on time changes may not be intuitively clear, it is easy to implement numerically. We just replace the uniform time interval $\Delta$ by $\tau$, based on the computation on each interest rate path.

\subsection{Discretization of the SDE System}

For the SABR-HW system, we also consider first the system without drift with time scale $\tau(t, \omega)$, and a low-bias Monte Carlo simulation scheme (Section 5.2, [11]). We then multiply the result by the exponentially integrated interest rate.

In this subsection, we describe the low-bias time discretization scheme to simulate the plain SABR system in the interest rate-dependent time scale $\tau(t, \omega)$ :

$$
\begin{aligned}
& d S(\tau)=\sigma(\tau) S(\tau)^{\beta} d W_{x}(\tau), \\
& d \sigma(\tau)=\gamma \sigma(\tau) d W_{\sigma}(\tau),
\end{aligned}
$$

where we denote $\tau(t, \omega)$ simply by $\tau$. Let us consider a system of three Brownian motions correlated with the following correlation matrix:

$$
\left(\begin{array}{ccc}
1 & \rho_{x, \sigma} & \rho_{x, r} \\
\rho_{x, \sigma} & 1 & 0 \\
\rho_{x, r} & 0 & 1
\end{array}\right) .
$$

Applying the Cholesky decomposition gives

$$
\begin{aligned}
& d W_{x}(\tau)=\rho_{x, \sigma} d W_{1}(\tau)+\rho_{x, r} d W_{2}(\tau)+\sqrt{1-\rho_{x, \sigma}^{2}-\rho_{x, r}^{2}} d U(\tau), \\
& d W_{\sigma}(\tau) \equiv d W_{1}(\tau) \\
& d W_{r}(\tau) \equiv d W_{2}(\tau)
\end{aligned}
$$

where the Brownian motions $W_{1}(\tau), W_{2}(\tau)$ and $U(\tau)$ are mutually independent.

Based on an argument by Schroder [29], we consider the invertible transformation of variables, $X(\tau)=S(\tau)^{1-\beta} /(1-\beta), \beta \neq 1$, so that

$$
d X(\tau)=\sigma(\tau) d W_{x}(\tau)-\frac{\beta \sigma(\tau)^{2}}{(2-2 \beta) X(\tau)} d \tau .
$$


We substitute Eqn. (5.4) into Eqn. (5.7) and integrate from 0 to $\tau(\Delta, \omega)$, which gives

$$
\begin{aligned}
X(\tau(\Delta, \omega))= & X(0, \omega)+\rho_{x, \sigma} \int_{0}^{\tau(\Delta, \omega)} \sigma(s) d W_{1}(s)+\rho_{x, r} \int_{0}^{\tau(\Delta, \omega)} \sigma(s) d W_{2}(s)- \\
& \int_{0}^{\tau(\Delta, \omega)} \frac{\beta \sigma(s)^{2}}{(2-2 \beta) X(s)} d s+\sqrt{1-\rho_{x, \sigma}^{2}-\rho_{x, r}^{2}} \int_{0}^{\tau(\Delta, \omega)} \sigma(s) d U(s) .
\end{aligned}
$$

In [11] it was shown that $\int_{0}^{\tau(\Delta, \omega)} \sigma(s) d W_{1}(s)=(\sigma(\tau(\Delta, \omega))-\sigma(0)) / \gamma$. It is also not difficult to show that $\int_{0}^{\tau(\Delta, \omega)} \sigma(s) d W_{2}(s)$ is a Gaussian integral with variance $\int_{0}^{\tau(\Delta, \omega)} \sigma(s)^{2} d s$, because of the independence of $W_{1}$ and $W_{2}$. Hence, we can replace the Gaussian integral:

$$
\int_{0}^{\tau(\Delta, \omega)} \sigma(s) d W_{2}(s)=\zeta(\tau(\Delta, \omega)) W_{2}(\tau(\Delta, \omega))
$$

where we have defined $\zeta(\tau(\Delta, \omega)):=\sqrt{\int_{0}^{\tau(\Delta, \omega)} \sigma(s)^{2} d s / \tau(\Delta, \omega)}$.

Based on these results, we can sample the SABR system without drift term and use the conditional scheme proposed in [11], conditional on the terminal volatility, the integrated volatility and the realization of $W_{2}$ :

$$
\begin{aligned}
X(\tau(\Delta, \omega))= & X(0)+\frac{\rho_{x, \sigma}}{\gamma}\{\sigma(\tau(\Delta, \omega))-\sigma(0)\}+\rho_{x, r} \zeta(\tau(\Delta, \omega)) W_{2}(\tau(\Delta, \omega))- \\
& \int_{0}^{\tau(\Delta, \omega)} \frac{\beta \sigma(s)^{2}}{(2-2 \beta) X(s)} d s+\sqrt{1-\rho_{x, \sigma}^{2}-\rho_{x, r}^{2}} \int_{0}^{\tau(\Delta, \omega)} \sigma(s) d U(s),
\end{aligned}
$$

where we have used (5.8).

Conditional on the volatility, the integrated variance and Brownian motion $W_{2}$, process $X$ is a shifted Bessel process, $\tilde{X}$, with dynamics:

$$
\begin{aligned}
d \tilde{X}(\tau) & :=\sqrt{1-\rho_{x, \sigma}^{2}-\rho_{x, r}^{2}} \sigma(\tau) d U(\tau)-\frac{\beta \sigma(\tau)^{2}}{(2-2 \beta) \tilde{X}(\tau)} d \tau, \\
\tilde{X}(0) & =X(0)+\frac{\rho_{x, \sigma}}{\gamma}\{\sigma(\tau(\Delta))-\sigma(0)\}+\rho_{x, r} \zeta(\tau(\Delta)) W_{2}(\tau(\Delta)) .
\end{aligned}
$$

We define another change of variables, $Y(\tau):=\tilde{X}(\tau)^{2}$, and apply Itô's lemma:

$$
\begin{aligned}
d Y(\tau) & =2 \tilde{X}(\tau) d \tilde{X}(\tau)+d \tilde{X}(\tau)^{2} \\
& =2 \tilde{X}(\tau)\left(\sqrt{1-\rho_{x, \sigma}^{2}-\rho_{x, r}^{2}} \sigma(\tau) d U(\tau)-\frac{\beta \sigma(\tau)^{2}}{(2-2 \beta) \tilde{X}(\tau)} d \tau\right)+\left(1-\rho_{x, \sigma}^{2}-\rho_{x, r}^{2}\right) \sigma(\tau)^{2} d \tau \\
& =2 \sqrt{Y(\tau)} \sqrt{1-\rho_{x, \sigma}^{2}-\rho_{x, r}^{2}} \sigma(\tau) d U(\tau)+ \\
& \left(\frac{1-2 \beta-\left(\rho_{x, \sigma}^{2}+\rho_{x, r}^{2}\right)(1-\beta)}{(1-\beta)\left(1-\rho_{x, \sigma}^{2}-\rho_{x, r}^{2}\right)}\right)\left(1-\rho_{x, \sigma}^{2}-\rho_{x, r}^{2}\right) \sigma(\tau)^{2} d \tau
\end{aligned}
$$

Let us define the time change, $v(\tau(\Delta, \omega))=\left(1-\rho_{x, \sigma}^{2}-\rho_{x, r}^{2}\right) \int_{0}^{\tau(\Delta, \omega)} \sigma(s)^{2} d s$.

Due to the independence of the Brownian motion, $U$, and the volatility process, a

Brownian motion under $\operatorname{clock} v(\tau(\cdot, \cdot))$ has the same distribution as $\sqrt{1-\rho_{x, \sigma}^{2}-\rho_{x, r}^{2}} \int_{0}^{\tau(\Delta, \omega)} \sigma(s) d U(s)$, i.e.

$$
U(v(\tau(\Delta, \omega))) \equiv \int_{0}^{v(\tau(\Delta, \omega))} d U_{s}=\sqrt{1-\rho_{x, \sigma}^{2}-\rho_{x, r}^{2}} \int_{0}^{\tau(\Delta, \omega)} \sigma(s) d U(s) .
$$

We substitute this time change into Eq. (5.9), and obtain:

$$
d Y(v(\tau))=2 \sqrt{Y(v(\tau))} d U(v(\tau))+\left(\frac{1-2 \beta-\left(\rho_{x, \sigma}^{2}+\rho_{x, r}^{2}\right)(1-\beta)}{(1-\beta)\left(1-\rho_{x, \sigma}^{2}-\rho_{x, r}^{2}\right)}\right) d v(\tau),
$$


which gives us a time changed squared Bessel process of dimension $\delta=\frac{1-2 \beta-\left(\rho_{x, \sigma}^{2}+\rho_{x, r}^{2}\right)(1-\beta)}{(1-\beta)\left(1-\rho_{x, \sigma}^{2}-\rho_{x, r}^{2}\right)}$ with initial value $Y(0)=\tilde{X}(0)^{2}$. The time change depends on the interest rate path via $\tau(\cdot, \cdot)$ and depends on the volatility path as a result of the time change function $v(\cdot)$. Each change of time is conditioned on specific path information of the volatility and the interest rate paths.

The stochastic volatility induced time change, $v(\cdot)$, can be computed by an asymptotic expansion, see [11] (Sect. 3.4). In order to determine the change of time scale $\tau(\cdot, \cdot)$, we have to compute integral $\int_{0}^{\Delta} r(s) d s$ in Eq. (5.3). There are several ways to approximate this integral. A straightforward approach is the following discrete approximation:

$$
\int_{0}^{\Delta} r(s) d s \approx \Delta\left[w_{1} r(0)+w_{2} r(\Delta)\right]
$$

The constants $w_{1}, w_{2}$ can be chosen in different ways, for example, in an Euler scheme we have $w_{1}=1, w_{2}=0$. A central discretization employs $w_{1}=w_{2}=\frac{1}{2}$. This latter scheme is computationally efficient and sufficiently accurate if the underlying stochastic process is slowly varying. This is the case for these interest rate processes. The calibrated interest rate process usually includes a volatility parameter $\eta<1 \%$.

The CDF of process $Y$ can be obtained by the properties of squared Bessel processes. By a mapping:

$$
h: s \rightarrow \frac{s^{2-2 \beta}}{(1-\beta)^{2}}, \quad s \geq 0
$$

with its inverse

$$
h^{-1}: y \rightarrow \frac{y^{2-2 \beta}}{(1-\beta)^{2}}, \quad y \geq 0
$$

we can define $S(\tau(\Delta, \omega))=h(Y(\nu(\tau(\Delta, \omega))))$ and $Y(0)=h^{-1}(S(0))=S(0)^{2(1-\beta)} /(1-\beta)^{2}$.

So, we have the following proposition:

Proposition 5.1 (Cumulative distribution for the conditional SABR process). The cumulative distribution for $S(\tau(\Delta, \omega))$, conditional on $\sigma(\tau(\Delta, \omega))$ and $\int_{0}^{\tau(\Delta, \omega)} \sigma_{S}^{2} d s$ with an absorbing boundary at $S=0$ reads

$$
\operatorname{Pr}[S(\tau(\Delta, \omega)) \leq x \mid S(0)]=1-\chi^{2}(a ; b, c),
$$

where

$$
\begin{aligned}
a & =\frac{1}{v(\tau(\Delta, \omega))}\left[\frac{S(0)^{1-\beta}}{(1-\beta)}+\frac{\rho}{\alpha}\{\sigma(\tau(\Delta, \omega))-\sigma(0)\}+\rho_{x, r} \cdot \zeta(\tau(\Delta, \omega)) W_{2}(\tau(\Delta, \omega))\right]^{2}, \\
b & =2-\frac{1-2 \beta-\left(\rho_{x, \sigma}^{2}+\rho_{x, r}^{2}\right)(1-\beta)}{(1-\beta)\left(1-\rho_{x, \sigma}^{2}-\rho_{x, r}^{2}\right)}, \\
c & =\frac{S(\tau(\Delta, \omega))^{2(1-\beta)}}{(1-\beta)^{2} v(\tau(\Delta, \omega))}, \\
v(\tau(\Delta, \omega)) & =\left(1-\rho^{2}\right) \int_{0}^{\tau(\Delta, \omega)} \sigma(s)^{2} d s .
\end{aligned}
$$

and $\chi^{2}(x ; \delta, \lambda)$ is the non-central chi square cumulative distribution function for random variable $x$ with non-centrality parameter $\lambda$ and degree of freedom $\delta$.

Proof: The proof proceeds along the lines of the work in Islah [21] and Chen [11]. 
Summary of the algorithm The algorithm to sample the SABR-HW system for a time interval $[0, \Delta]$ consists of the following steps:

1. Draw samples of $r(\Delta)$ from a normal distribution with mean and variance defined by Eq. (2.8) and (2.9), respectively;

2. Apply a drift interpolation $\int_{0}^{\Delta} r(s) d s \approx \Delta\left(\frac{1}{2} r(0)+\frac{1}{2} r(\Delta)\right)$, for the quantity in Formula (5.3) for the pathwise (stochastic interest rate induced) rescaled time step $\tau(\Delta, \omega)$;

3. Conditional on $\sigma(\tau(\Delta, \omega))$, draw samples of $\int_{0}^{\tau(\Delta, \omega)} \sigma(s)^{2} d s$ by the method proposed in Section 3.4 of [11];

4. Conditional on $\sigma(\tau(\Delta, \omega))$ and $\int_{0}^{\tau(\Delta, \omega)} \sigma(s)^{2} d s$, draw samples of the dynamics without drift, $S$, in the time scale $\tau$ from the non-central chi square distribution, described in Prop. 5.1;

5. Find the asset price process with drift, $S^{(r)}(\tau(\Delta, \omega))$, by multiplying the estimated $\int_{0}^{\Delta} r(s) d s$ (Step 2) with $S(\tau(\Delta, \omega))$.

\subsubsection{Numerical Experiment with European Options}

We consider the pricing of European options in the SABR-HW model by the lowbias Monte Carlo method. We focus on a call option, maturing at time $T$ with strike price $K$, and denote the exact option price at initial time by $C(K, 0)$. It is approximated by:

$$
\hat{C}(K, 0)=\mathbb{E}\left[(S(T)-K)^{+}\right] .
$$

$\hat{C}(K, 0)$ is typically not equal to $C(K, 0)$, and we define the bias, $e$, of the simulation as a function of the time step, $\Delta$, and analyze its behavior.

We will use the parameter sets I, II and III in Table 3.1 to carry out the numerical experiments for the call options with $T=10$ and ATM strikes. As the benchmark, we apply a Monte Carlo simulation based on the Euler discretization with very many time steps, 400 time steps per year, and report the average of three runs as the reference prices. For Sets I, II and III, the option prices obtained by the low-bias Monte Carlo method are listed in Table 5.1 for time steps $\Delta$ ranging from 1 to 32 steps a year.

We plot the differences between the MC estimate and the reference values for different step sizes in Figure 5.1, from which we see that the low-bias scheme is advantageous to the Euler scheme with respect to its low bias. By increasing the number of time steps the low-bias scheme produces smaller errors than the Euler scheme for all parameter settings.

\subsubsection{Time-Dependent Parameters}

We can also discretize System (2.1) with the low-bias scheme by assuming that the functions can be approximated by piecewise constant functions on $[t, t+\Delta]$ with value $\bar{\gamma}$. According to the arguments by Andersen [1] and Glasserman [15] (pp. 130) we then use $\bar{\gamma}=(\gamma(t)+\gamma(t+\Delta)) / 2$, which leads to a modification of System (2.1) that can easily be simulated by the low-bias scheme. More precisely, the volatility process in the dynamical SABR-HW system is sampled by the following formula

$$
\Sigma(t+\Delta)=\bar{g} \cdot k(t) \cdot \exp \left(-\frac{1}{2} \bar{h}^{2} \Delta+\bar{h} Z \sqrt{\Delta}\right), k(0)=1,
$$

where

$$
\bar{g}=\frac{g(0, t)+g(0, t+\Delta)}{2}, \bar{h}=\frac{h(0, t)+h(0, t+\Delta)}{2} .
$$

As a result, the formula for the integrated variance has to be adapted as well: it has to be multiplied by the factor $\bar{g}^{2}$. 

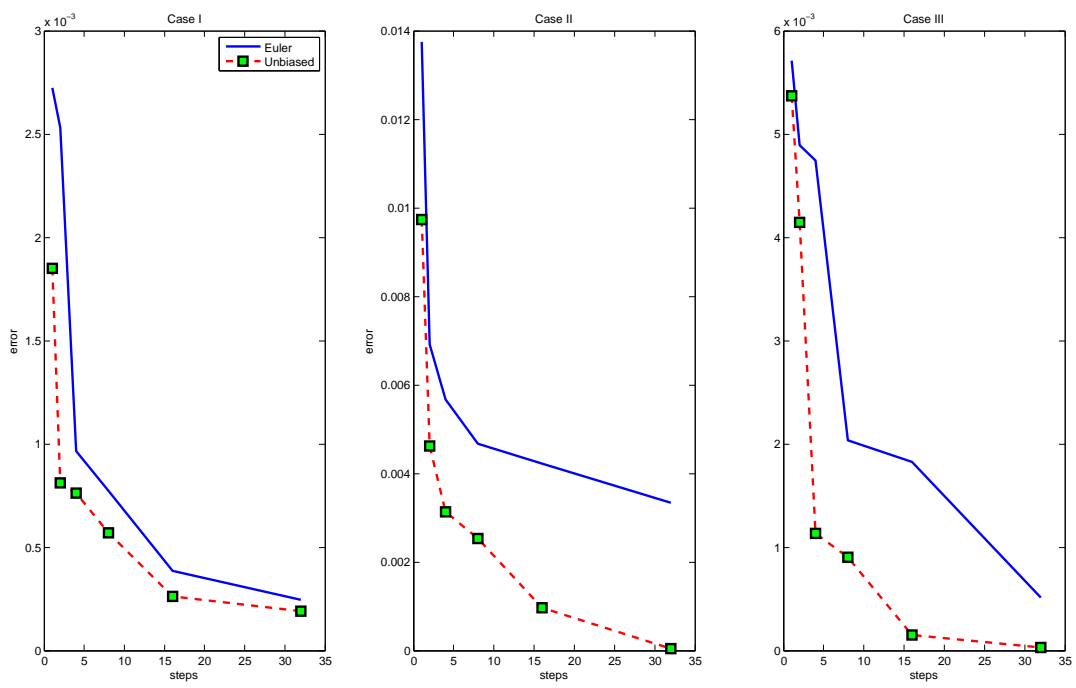

Figure 5.1: The MC error as a function of the number of time steps. Note the different scaling of the three figures.

Table 5.1: Estimated 10 year ATM call option prices for Cases I, II and III.

\begin{tabular}{c|cccccc}
\hline \hline & \multicolumn{2}{|c}{ Case I } & \multicolumn{2}{c}{ Case II } & \multicolumn{2}{c}{ Case III } \\
\hline & \multicolumn{5}{c}{$\mathrm{T}=10$} \\
\hline$\Delta$ & Low-bias & Euler & Low-bias & Euler & Low-bias & Euler \\
1 & 0.4653 & 0.4699 & 0.4731 & 0.4771 & 0.5485 & 0.5374 \\
$1 / 2$ & 0.4680 & 0.4697 & 0.4680 & 0.4703 & 0.5473 & 0.5382 \\
$1 / 4$ & 0.4664 & 0.4662 & 0.4665 & 0.4691 & 0.5420 & 0.5479 \\
$1 / 8$ & 0.4666 & 0.4664 & 0.4659 & 0.4681 & 0.5440 & 0.5451 \\
$1 / 16$ & 0.4669 & 0.4675 & 0.4644 & 0.4676 & 0.5430 & 0.5449 \\
$1 / 32$ & 0.4669 & 0.4673 & 0.4633 & 0.4667 & 0.5431 & 0.5432 \\
\hline Reference & 0.4671 & \multicolumn{7}{c}{0.4634} & & 0.5431 & \\
\hline
\end{tabular}




\section{Conclusion}

In this article we have presented the dynamical SABR-HW model as an alternative model for pricing long-maturity equity options and equity-interest rate hybrid products. We have defined the model, introduced its building blocks and described several issues for the practical application of the SABR-HW model, like model calibration and option pricing. At several places we have presented numerical techniques that are not commonly used by the financial industry, like the low-bias discretization scheme and a weighted Monte Carlo technique to enhance the calibration.

In particular, we have proposed an invertible projection formula for the constant parameter SABR-HW model connecting it to the plain SABR model. The basis for this projection was a change of measure, to the $T$-forward measure, and a linearization. The projection formula greatly simplifies the calibration of the SABR-HW model.

The inversion of the projection formula serves as a first step in the calibration procedure, i.e. it gives a rapid and fairly accurate approximation of the constant SABRHW parameters at each maturity. Based on these parameters, we have defined timedependent functions in the dynamical SABR-HW model, that are consistent with the market implied volatilities for all maturities.

In the final calibration step, non-uniform Monte Carlo weights have been determined in such a way that the implied volatilities from the market and those generated by the Monte Carlo paths of SABR-HW model match optimally. The overall calibration procedure is highly efficient and accurate.

Exotic contracts have then been priced using the weighted Monte Carlo paths generated by a low-bias time discretization scheme of the dynamical SABR-HW model. An advantage of the low-bias scheme is that accurate Monte Carlo results can be obtained for large time steps. This is particularly useful when long-maturity options are considered.

\section{References}

[1] L. Andersen. Simple and efficient simulation of the Heston stochastic volatility model. J. Comp. Finance, 11(3), 1-42, 2008.

[2] M. Avellaneda and P. Jäckel. Weighted Monte Carlo, Encyclopedia of Quantitative Finance. John Wiley and Sons, first edition, 2010.

[3] M. Avellaneda, C. Friedman, R. Holmes, and D. Samperi. Calibrating volatility surfaces via relative-entropy minimization. Appl. Math. Finance, 4: 37-64, 1997.

[4] M. Avellaneda. Minimum-relative-entropy calibration of asset-pricing models. Int. J. Theoret. Appl. Finance, 1: 447-472, 1998.

[5] M. Avellaneda, R. Buff, C. Friedman, N. Grandechamp, L. Kruk, and J. Newman. Weighted Monte Carlo: A new technique for calibrating asset-pricing models. Int. J. Theoret. Appl. Finance, 4: 91-119, 2000.

[6] D. Brigo and F. Mercurio. Interest rate models - Theory and practice: with smile, inflation and credit. Springer Finance, second edition, 2007.

[7] M. Broadie and Ö. Kaya. Exact simulation of stochastic volatility and other affine jump diffusion processes. Operations Research, 54: 217-231, 2006.

[8] S. Boyd and L. Vandenberghe. Convex Optimization. Cambridge Univ. Press, Cambridge, U.K., 2004.

[9] P. W. Buchen and M. Kelly. The maximum entropy distribution of an asset inferred from option prices. J. Fin. Quantit. Analysis, 31: 143-159, 1996. 
[10] B. Chen and C.W. Oosterlee and S. van Weeren. Analytic approximation to constant maturity swap convexity correction in a multi-factor SABR model. Int. J. Theoret. Appl. Finance, 13(7):1019-1046, 2010.

[11] B. Chen, C.W. Oosterlee, and J.A.M. van der Weide. An efficient unbiased simulation scheme for SABR stochastic volatility model. CWI Working Paper, 2010, to appear in Int. J. Theoret. Appl. Finance.

[12] L.A. Grzelak and C.W. Oosterlee On the Heston model with stochastic interest rates SIAM J. Fin. Math.

[13] J. Gatheral. The volatility surface: A practitioner's guide. Wiley, New York, 2006.

[14] H. Geman, N. El Karoui, and J.C. Rochet. Changes of numéraire, changes of probability measures and pricing of options. J. Appl. Probability, 32: 443-458, 1996.

[15] P. Glasserman. Monte Carlo methods in financial engineering. Springer, New York, 2004.

[16] D. Goldenberg. A unified method for pricing options on diffusion processes. J. Fin. Economics, 29: 3-34, 1991.

[17] P.S. Hagan. Adjusters: Turning good prices into great prices. Wilmott Magazine, 56-59, December 2002.

[18] P.S. Hagan, D. Kumar, A.S. Lesniewski, and D.E. Woodward. Managing smile risk. Wilmott Magazine, 84-108, November 2002.

[19] J. Hull and A. White. Using Hull-White interest rate trees. J. Derivatives, 3(3):26-36, 1996.

[20] C. Hunter and G. Picot, Hybrid derivatives. The euromoney derivatives and risk management handbook. BNP Paribas, 2005.

[21] O. Islah. Solving SABR in Exact Form and Unifying it with LIBOR Market Model. SSRN eLibrary, 2009.

[22] T. Kariya and H. Kurata. Generalized Least Squares. Wiley, New York, 2004.

[23] R. Mendoza, P. Carr, and V. Linetsky. Time changed Markov processes in unified credit-equity modelling. Math. Finance, 20(4): 527-569, 2010.

[24] V. Piterbarg. Smiling hybrids. Risk, 19: 66-71, 2006.

[25] R. Rebonato. Modern pricing of interest-rate derivatives. Princeton University Press, first edition, 2002.

[26] R. Rebonato. A time-homogeneous, SABR-consistent extension of the LMM. Risk, 20: 92-97, 2006.

[27] R. Rebonato and R. White. Linking caplet and swaption prices in the LMM-SABR model. http://www.riccardorebonato.co.uk/papers/LMMSABRSwaption.pdf, 2007.

[28] R. Rebonato. The SABR/LIBOR Market Model: Pricing, calibration and hedging for complex interest-rate derivatives. John Wiley and Sons, first edition, 2009.

[29] M. Schroder. Computing the constant elasticity of variance option pricing formula. J. Finance, 44: 211-219, 1989.

[30] G. West. Calibration of the SABR model in illiquid markets. Appl. Math. Finance, 12(4): 371-385, 2005.

[31] P. Wilmott. Cliquet options and volatility models. Wilmott Magazine, 78-83, March 2002. 